\title{
Complex physical rehabilitation of patients with chronic obstructive pulmonary disease at a polyclinic stage of treatment
}

\author{
DOI: https://doi.org/10.5114/pq.2019.84268
}

\author{
Larysa Ruban', Maryna Kochuieva², Anton Rohozhyn², Gennadii Kochuiev², Hanna Tymchenko², Yana Samburg² \\ ${ }^{1}$ Kharkov State Academy of Physical Culture, Kharkov, Ukraine \\ ${ }^{2}$ Kharkiv Medical Academy of Postgraduate Education, Kharkov, Ukraine
}

Abstract

Introduction. The study was to assess the effectiveness of a comprehensive physical rehabilitation program based on the dynamics of the functional parameters of the respiratory and cardiovascular systems, and the tolerance of daily stress in middle-aged patients with chronic obstructive pulmonary disease (COPD). The incidence of COPD continues to grow, and life expectancy of the patients is shortened by an average of 8 years. Globally, losses from COPD are projected to increase over the coming decades owing to the long-term exposure to risk factors and population aging. A significant role in improving COPD patients condition and preventing the disease is played by physical rehabilitation.

Methods. Analysis of modern scientific literature, content analysis of outpatient documentation, conclusions of X-ray examinations, anthropometric measurements, pulse oximetry, arterial tonometry, spirographic studies, 6-minute walk distance, methods of mathematical statistics.

Results. A comprehensive physical rehabilitation program was developed for use in a polyclinic, which included a common set of physical exercises combined with breathing gymnastics with elements of superficial breathing, sound gymnastics, therapeutic massage, nebulizer therapy, Nordic walking. After the program application, chest excursion increased and airway patency statistically significantly improved. The exercise tolerance of the cardiovascular system increased.

Conclusions. The physical rehabilitation program for middle-aged COPD patients contributed to the economization of the respiratory system, slowing down the recovery of cardiovascular parameters, and improving the tolerance of daily exercise, which was confirmed by mathematical processing.

Key words: COPD, physical rehabilitation, respiratory system, cardiovascular system, daily exercise

\section{Introduction}

According to World Health Organization (WHO) forecasts, the incidence of chronic obstructive pulmonary disease (COPD) in 2020 will take $5^{\text {th }}$ place in the world among all diseases, and by 2030 COPD will constitute the $4^{\text {th }}$ cause of total mortality. The incidence of COPD continues to grow steadily, and the life expectancy of patients is shortened by an average of 8 years, whereas recently a decrease has been observed in the rate of mortality from myocardial infarction, tuberculosis, and oncological diseases [1-5]. In Ukraine, approximately 3 million people had a diagnosis of COPD in 2015 [6].

There are a number of reasons for the increase in the incidence and prevalence of COPD, including environmental pollution or progressive aging of the population; the most significant factor, however, remains smoking. WHO estimates that about $1 / 3$ of the world's adult population are smokers. In our country, $58 \%$ of men and $14 \%$ of women consider themselves to be smokers. The prevalence of smoking among adolescents aged 13-16 is striking - it already reaches $50 \%$. According to the British Enhanced Resource Company, Ukraine ranks $2^{\text {nd }}$ in the world in the number of cigarettes smoked per person per year. On average, a Ukrainian smokes 2500 cigarettes per year, or 7 pieces per day. Taking into account the demographic situation in our country associated with the aging of the population, the growing number of smokers, as well as the deterioration of the environment, we can most likely assume an increase in morbidity and mortality from COPD in Ukraine. Globally, losses from COPD are projected to increase over the coming decades owing to the long-term exposure to COPD risk factors and population aging [7-10].

A significant role in improving the condition of patients with COPD and preventing this disease is played by physical rehabilitation, including medical physical culture, massage, physiotherapy, or diet. These help patients to get rid of bad habits, in particular, smoking, improve the respiratory and cardiovascular systems condition, strengthen the muscular system, increase immunoreactivity. So far, the need for a physical rehabilitation appointment in COPD has not been sufficiently substantiated, which has led to the relevance of this work and the need to develop a comprehensive physical rehabilitation program for patients in the clinic $[8,9]$.

The purpose of the study was to evaluate the effectiveness of a comprehensive program of physical rehabilitation on the basis of the dynamics of the respiratory and cardiovascular system functional parameters among middleaged patients with COPD.

Correspondence address: Larysa Anatolyevna Ruban, Department of Sports Medicine, Biochemistry and Anatomy, Kharkov State Academy of Physical Culture, Klochkovska Street 99, Kharkov, 61058, Ukraine, e-mail: slarisaruban@gmail.com

Received: 21.11.2018

Accepted: 18.02 .2019

Citation: Ruban L, Kochuieva M, Rohozhyn A, Kochuiev G, Tymchenko H, Samburg Y. Complex physical rehabilitation of patients with chronic obstructive pulmonary disease at a polyclinic stage of treatment. Physiother Quart. 2019;27(2):11-16; doi: https://doi.org/10.5114/ pq.2019.84268. 


\section{Subjects and methods}

\section{Participants}

The study was conducted in the conditions of the Department of Physical Therapy at the Kharkov City Polyclinic No. 8. The study included 52 male patients diagnosed with COPD, stage IIB of severity, in remission. They were randomly divided into 2 groups. The main group (MG) consisted of 35 patients, who along with the standard drug therapy were engaged in a comprehensive program of physical rehabilitation developed by the authors. The control group (CG) involved 17 patients with a similar diagnosis of COPD, who received standard drug therapy and were engaged in the regular physiotherapy program at the Department. The groups were balanced with regard to sex and age; $78 \%$ of subjects in both groups were smokers.

\section{Intervention}

Classes with surveyed contingents of $M G$ and $C G$ were performed by group method 4 times a week, with the course of treatment lasting 28 days.

In the procedure of medical gymnastics, CG performed general exercises for muscles of the upper shoulder girdle, trunk, lower limbs, alternating with breathing exercises. The alternation of respiratory and general developmental exercises was $2: 1$, followed by $3: 1$ in the initial position sitting and standing, the rate of execution was slow with a maximum amplitude. The number of exercise repetitions was $8-10$, with the procedure duration of 30-40 minutes. Shallow breast technique was applied during the program construction. The mechanism of action of superficial respiration consists in the fact that owing to the peculiarity of this type of respiration, the air during inhalation and exhalation passes only in the upper respiratory tract, which leads to the accumulation of carbon dioxide in the blood and reflex stimulation of the respiratory centre; this in turn causes the expansion of the bronchial trees and improves the passage of the respiratory tract. Independent classes included acoustic gymnastics, which extends the exhalation phase and facilitates the relaxation of spasmodic bronchi and bronchioles. At exhalation, various sounds are pronounced at a slow pace, with an average amplitude, without forcing the breath. Depending on the period, exhalation lasted 6-15 seconds. After 2 weeks, the patients were supplemented with the following exercises: breathing in the corners of the mouth, breathing alternately with each corner of the mouth, breathing through the clenched nose, breathing alternately with each nostril. The number of exercises, dosage, and duration were determined individually.

The prescribed massage of the lower and upper extremities was aimed to improve blood and lymph flow and eliminate congestion on the periphery, stimulating the rush of venous blood to the heart. The duration of the massage was 20 minutes. With physiotherapeutic agents, inhalations were administered with Decasan via a nebulizer. The advantages of inhalation through a nebulizer are as follows: the inhalation technique is easy to perform since it does not require a forced manoeuver; more often, coordination of inhalation and inhalation of the drug is not necessary; there is a possibility to administer a high dose of a drug; drugs are sprayed through a nebulizer; there are no freons or other propellants in the solutions. Long-term and regular use of nebulizer therapy leads to an improvement in mucociliary clearance and bronchial patency, and a decrease in the frequency and severity of asthma attacks. The treatment regimen constituted in 1 inhalation per day using 5-10 ml of Decasan, with a course of 10 treatments.

The comprehensive program of physical rehabilitation for MG patients included Nordic walking [11, 12]. Given the active work of the entire body while walking, the respiratory volume of the lungs increases by about $30 \%$. To date, it has been proven that this progressive walking is $46 \%$ more effective than normal walking or jogging and contributes to the normalization of vascular tone [5, 8]. CG patients passed a distance of 1500-2500 meters per day on a flat terrain, with a breathing rhythm of inhalation in 2-3 steps and exhalation in 5-6 steps.

Patients in CG were treated with physiotherapy 4 times a week, in accordance with the generally accepted method, in an exercise room. They performed general development exercises with the control of the act of respiration, and static and dynamic breathing exercises. CG patients received therapeutic massage 5 times a week, with the classical technique: they started the procedure from the back of the body, after which the finger pads were thoroughly rubbed between the intercostal intervals and the area of the hypochondrium and the edge of the palm - under the shoulder blade. At the end of the massage, light tapping was performed all over the surface, and stroking was applied. Therapeutic percussion of the chest was carried out with draining in minute cycles with a frequency of 40-60 beats per minute and a subsequent pause (1 minute), for 15 minutes [13]. Alkaline inhalations with bronchodilators, a course of 8-10 procedures, were used with physiotherapeutic means for the dilution and sputum discharge.

Among the participants in both groups, $78 \%$ were smokers. Therefore, as one of the main tasks in the training of patients with COPD, we proposed smoking cessation. When working with patients, in individual communication, with the help of visual aids, we explained the structure of the lungs and bronchi, the causes and consequences of COPD, as well as the harm from smoking and pollutants of the environment.

\section{Assessment}

To evaluate the effectiveness of the developed program of physical rehabilitation, the following research methods were applied: analysis of modern scientific literature, content analysis of outpatient documentation, conclusions of X-ray examinations, anthropometric measurements, pulse oximetry, arterial tonometry, spirographic studies, and 6-minute walk distance (6MWD).

Spirographic studies were carried out with the use of a Spirolab computer system (produced by the KhAl Medica Research Institute, Kharkov). The parameters of the lung ventilation function were analysed with the Pistelli norms (1986). Selected features of the respiratory system that characterize its functional and reserve capabilities were considered: respiratory rate $(R R)$, respiratory volume (RV), minute volume of respiration (MVR), vital capacity (VC), forced vital capacity (FVC), maximal ventilation of the lungs $(\mathrm{MVL})$, volume of forced exhalation in the $1^{\text {st }}$ second $\left(\mathrm{VFE}_{1}\right)$, percentage ratio of $\mathrm{VFE}_{1}$ to $\mathrm{FVC}$, peak expiratory flow (PEF), maximum expiratory flow at the level of $25-50-75 \%$ FVC $\left(\mathrm{MEF}_{25}, \mathrm{MEF}_{50}, \mathrm{MEF}_{75}\right)$.

With the help of hypoxic Stange and Genchi samples, in accordance with the generally accepted method, the body's resistance to hypoxia and hypercapnia was studied. To assess the cardiovascular system function, pulse oxim- 
etry (measurement of heart rate with the palpation method for 10 seconds, 3 times until a stable figure was obtained after 2-3 minutes of rest, with recalculation for 1 minute) and arterial tonometry (with systolic [BPs], diastolic [BPd], and pulse $[\mathrm{BPp}]$ blood pressure determination) were performed.

The 6MWD was used to detect daily load tolerance. The distance in meters was measured for 6 minutes and compared with the proper 6MWD, calculated with the formula considering age, body weight, and height [9].

\section{Statistical analysis}

Statistical processing was performed with the use of the general-purpose data package of Statistica for Windows version 6.0. The data are presented in the form of an arithmetic mean $(\bar{x})$, its error $(m)$, Student's reliability criterion $(t)$, degree of reliability $(p)$. For all the analyses conducted, the differences were considered reliable at the significance level of $p<0.05$, where the minimum reliability of the differences was $95 \%$. The empirical data were tested with the $W$ criterion of Shapiro-Wilk and it was concluded that the data in our sample corresponded to a normal distribution at the 0.05 significance level.

\section{Ethical approval}

The research related to human use has been complied with all the relevant national regulations and institutional policies, has followed the tenets of the Declaration of Helsinki, and has been approved by the authors' institutional review board or an equivalent committee.

\section{Informed consent}

Informed consent has been obtained from all individuals included in this study.

\section{Results}

In the content analysis of outpatient charts for lung X-ray examination in COPD stage IIB patients, only $2 \%$ of the individuals showed no changes in severity, $11 \%$ had emphysematous pulmonary fields with moderate signs of pneumosclerosis, $13 \%$ presented with high standing and sealing of the diaphragm dome due to fibrous pleural adhesions, and in $74 \%$ there were pulmonary fields without infiltrative changes. Structural roots and compensatory basal emphysema were observed. With clinical evidence, chronic bronchitis could not be ruled out.

As a result of evaluating the indicators of physical development, in accordance with the measurements of height and weight, no statistically significant differences in CG and MG patients as for the magnitude of chest excursions were revealed in the primary examination $(p>0.05)$.

The analysis of external respiration indicators, characterizing the volumetric parameters of the lungs, pulmonary ventilation, reserve respiratory system, resistance to hypoxia, and bronchial and aerobic performance, did not determine statistically significant differences between the groups during the primary examination $(p>0.05)$ (Table 1$)$.

All these changes indicate the presence of obstruction and a decrease in lung tissue elasticity, that is, restrictive changes.

With regard to changes in the function of the cardiovascular system during the initial examination in both groups, there was approximately the same heart rate value, BPs, BPd, $\mathrm{BPp}$ at rest; recovery was noted after deceleration of these indicators of physical activity. The values of physical load tolerance, according to the 6MWD test, were reduced: 512.13 $\pm 13.10 \mathrm{~m}$ in MG patients, $532.18 \pm 12.87 \mathrm{~m}$ in CG patients, in comparison with the proper values (calculated as 700 $852 \mathrm{~m}$ and 719-872 m for MG and CG patients, respectively). When assessing the type of cardiovascular system response to the load, in $5 \mathrm{MG}$ patients and $6 \mathrm{CG}$ patients, a normotonic type of reaction to physical activity was ob-

Table 1. Respiratory function indicators in patients of both groups during the initial examination $(\bar{x} \pm m)$

\begin{tabular}{|c|c|c|c|c|c|c|}
\hline No. & Indicators & $\mathrm{MG}(n=35)$ & $t_{1}$ & $\mathrm{CG}(n=17)$ & $t_{2}$ & Norm \\
\hline 1. & RR (per minute) & $18.58 \pm 0.28$ & 0.09 & $18.24 \pm 0.25$ & 0.06 & $16-18$ \\
\hline 2. & $\mathrm{RV}(\mathrm{I})$ & $0.59 \pm 0.02$ & 0.58 & $0.66 \pm 0.09$ & 0.48 & $0.3-0.8$ \\
\hline 3. & MVR (I) & $14.60 \pm 0.92^{*}$ & 7.58 & $13.98 \pm 0.27^{*}$ & 6.58 & $5-6$ \\
\hline 4. & VC (\%) & $70.29 \pm 2.68^{*}$ & 4.61 & $71.72 \pm 1.44^{*}$ & 4.89 & 87 \\
\hline 5. & FVC (\%) & $63.57 \pm 2.16^{*}$ & 7.62 & $64.28 \pm 2.29^{*}$ & 7.82 & 88 \\
\hline 6. & $\operatorname{VFE}_{1}(\%)$ & $62.24 \pm 2.37^{*}$ & 7.51 & $63.19 \pm 2.18^{*}$ & 7.69 & 87 \\
\hline 7. & $\mathrm{VFE}_{1} / \mathrm{FVC}(\%)$ & $65.05 \pm 2.47^{*}$ & 7.42 & $67.05 \pm 1.29^{*}$ & 7.22 & 90 \\
\hline 8. & PEF (\%) & $61.58 \pm 1.81^{*}$ & 7.15 & $63.51 \pm 1.81^{*}$ & 7.09 & 84 \\
\hline 9. & $\mathrm{MEF}_{25}(\%)$ & $63.16 \pm 1.41^{*}$ & 5.94 & $64.41 \pm 1.75^{\star}$ & 4.98 & 5.94 \\
\hline 10. & $\mathrm{MEF}_{50}(\%)$ & $54.37 \pm 1.91^{*}$ & 8.42 & $56.62 \pm 2.19^{*}$ & 8.56 & 8.42 \\
\hline 11. & $\mathrm{MEF}_{75}(\%)$ & $42.31 \pm 2.18^{*}$ & 9.06 & $45.43 \pm 2.81^{*}$ & 9.12 & 9.06 \\
\hline 12. & MVL (I/min) & $65.12 \pm 4.80^{*}$ & 10.09 & $61.18 \pm 4.21^{*}$ & 10.11 & 10.09 \\
\hline 13. & Stange sample (s) & $28.89 \pm 2.26^{*}$ & 6.55 & $32.54 \pm 2.19^{*}$ & 6.35 & 6.55 \\
\hline 14. & Genchi sample (s) & $18.36 \pm 1.20$ & 1.46 & $21.21 \pm 1.14$ & 1.32 & 1.46 \\
\hline
\end{tabular}

statistically significant difference as compared with norm $(p<0.05)$

$M G$ - main group, $C G$ - control group, $t_{1}$ - statistically significant difference in $M G(p<0.05), t_{2}$ - statistically significant difference in CG $(p>0.05), R R$ - respiratory rate, RV - respiratory volume, MVR - minute volume of respiration, VC - vital capacity, FVC - forced vital capacity, $\mathrm{VFE}_{1}$ - volume of forced exhalation in the $1^{\text {st }}$ second, $\mathrm{PEF}$ - peak expiratory flow, $\mathrm{MEF}_{25}, \mathrm{MEF}_{50}, \mathrm{MEF}_{75}-$ maximum expiratory flow at the level of $25-50-75 \%$ FVC, MVL - maximal ventilation of the lungs 
Table 2. Dynamics of chest excursion in patients of both groups $(\bar{x} \pm m)$

\begin{tabular}{|l|c|c|c|c|c|c|}
\hline Indicator & Stage & $\mathrm{MG}(n=35)$ & $\mathrm{CG}(n=17)$ & $t_{1}$ & $t_{2}$ & $t_{3}$ \\
\hline \multirow{2}{*}{ Chest excursion $(\mathrm{cm})$} & 1 & $2.86 \pm 0.05$ & $2.91 \pm 0.09$ & \multirow{2}{*}{7.76} & 1.65 & 5.91 \\
\cline { 2 - 5 } & 2 & $4.31 \pm 0.18$ & $3.12 \pm 0.09$ & & & \\
\hline
\end{tabular}

MG - main group, CG - control group, $t_{1}$ - statistically significant difference in MG $(p<0.05), t_{2}$ - statistically significant difference in CG $(p>0.05), t_{3}$ - statistically significant difference between MG and CG $(p<0.05)$

served. Among atypical reactions, hypertonic (12 MG subjects and 5 CG subjects) and hypotonic ones (10 MG participants and 4 CG participants) were most common; dystonic response was noted in $8 \mathrm{MG}$ patients and $2 \mathrm{CG}$ patients.

Thus, during the initial examination of the cardiovascular system in patients with COPD stage IIB, the economization of heart activity was violated at rest and during exercise, and a decrease in exercise tolerance was observed.

To assess the effectiveness of the developed comprehensive program of physical rehabilitation, a comparative analysis of the respiratory and cardiovascular systems and of the adaptation of the body to the daily load was conducted among patients from MG and CG.

Among MG participants, in $77 \%$ of cases, no shortness of breath was observed during the day at physical exertion and at rest, and isolated shortness of breath was noted in those who stopped using shallow breathing and did not require the use of inhaled bronchodilators (33\%). In MG patients, the excursion of the chest showed a statistically significant difference compared with the primary examination $(p<0.05)$; in CG subjects, chest excursion increased, but the change did not achieve statistical significance $(p>0.05)$ (Table 2).

We associate the statistically significant dynamics in $M G$ with the practice of Nordic walking within the physical rehabilitation program, by which the back is straightened, the posture is corrected, and the thorax is revealed.

When analysing the indices of respiratory function in $\mathrm{MG}$ patients after repeated examination, a statistically significant decrease in RR to $16.80 \pm 0.80$ respiratory movements per minute was revealed $(t=3.71 ; p<0.05)$, as well as a decrease in the value of MVR to $11.35 \pm 0.77$ I $(t=2.71 ; p<0.05)$. All this points at some development of the economization of the respiratory system activity in MG participants. In CG patients, positive dynamics was observed, but not reaching statistical significance $(p>0.05)$.

The dynamics of the VC indicator in MG patients statistically significantly increased $(p<0.05)$. CG subjects showed an improvement, but did not obtain statistically significant changes $(p>0.05)$ (Figure 1).

In the analysis of the dynamics of bronchial obstruction indicators, in MG patients, the values of $\mathrm{FVC}, \mathrm{VFE}_{1}$, and $\mathrm{VFE}_{1} / \mathrm{FVC}$ statistically significantly increased: 1.12 times $(t=$ 2.39), 1.11 times $(t=2.02)$, and 1.10 times $(t=2.18)$, respectively $(p<0.05)$; in CG patients, they increased 1.02 times

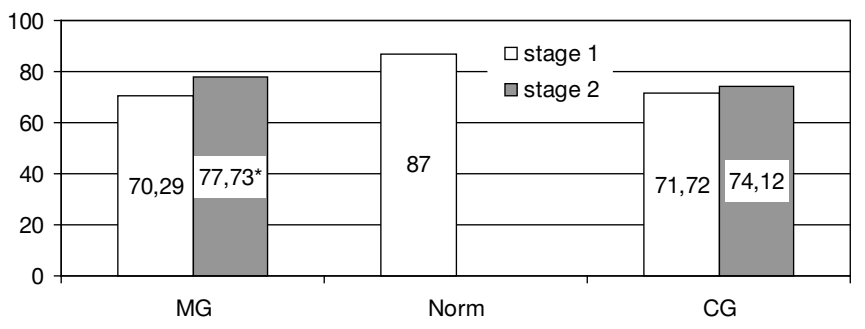

* statistically significant difference in MG $(t=2.16 ; p<0.05)$ $M G$ - main group, $C G$ - control group
Table 3. Dynamics of bronchial obstruction indicators in the studied COPD patients

\begin{tabular}{|l|c|c|c|}
\hline \multirow{2}{*}{ Groups } & \multicolumn{3}{|c|}{ Parameters } \\
\cline { 2 - 4 } & FVC & VFE1 & VFE1/FVC \\
\hline MG (stage 1) & 63.57 & 62.24 & 65.05 \\
\hline MG (stage 2) & $72.52^{*}$ & 69.18 & 72.18 \\
\hline CG (stage 1) & 64.28 & 63.19 & 67.05 \\
\hline CG (stage 2) & 66.72 & 65.12 & 68.67 \\
\hline
\end{tabular}

* statistically significant difference in $M G(t=2.39 ; p<0.05)$ $M G$ - main group, $C G$ - control group, FVC - forced vital capacity, $\mathrm{VFE}_{1}-$ volume of forced exhalation in the $1^{\text {st }}$ second

Table 4. Dynamics of speed indices of bronchial patency in the studied COPD patients

\begin{tabular}{|l|c|c|c|c|}
\hline \multirow{2}{*}{ Groups } & \multicolumn{4}{|c|}{ Parameters } \\
\cline { 2 - 5 } & PEF & MEF25 & MEF50 & MEF75 \\
\hline MG (stage 1) & 61.58 & 63.16 & 54.37 & 42.31 \\
\hline MG (stage 2) & 70.34 & 68.75 & 60.12 & 48.87 \\
\hline CG (stage 1) & 63.51 & 64.41 & 56.62 & 45.43 \\
\hline CG (stage 2) & 65.43 & 65.87 & 58.47 & 49.14 \\
\hline
\end{tabular}

MG - main group, CG - control group, PEF - peak expiratory flow, $\mathrm{MEF}_{25}, \mathrm{MEF}_{50}, \mathrm{MEF}_{75}$ - maximum expiratory flow at the level of $25-50-75 \%$ FVC

$(t=1.41), 1.03$ times $(t=0.72)$, and 1.02 times, respectively $(p>0.05)$ (Table 3$)$.

As for the dynamics of the speed indicators characterizing airway patency, in MG patients, the PEF parameter increased statistically significantly 1.14 times $(t=3.29 ; p<0.05)$, $\mathrm{MEF}_{25}$ increased 1.08 times $(t=2.58 ; p<0.05), \mathrm{MEF}_{50}$ increased 1.10 times $(t=2.20 ; p<0.05), \mathrm{MEF}_{75}$ increased 1.15 times $(t=2.09 ; p<0.05)$. In CG participants, these indicators tended to increase, but the change did not reach statistical significance $(p>0.05)$ : PEF increased 1.03 times $(t=$ $0.88), M_{25}$ increased 1.02 times $(t=0.69), M_{50}$ increased 1.03 times $(t=0.73), M F_{75}$ increased 1.08 times $(t=1.20)$ (Table 4).

In the medical observation in accordance with the results of the Stange and Genchi tests in MG and CG patients, the change of the body resistance to hypoxia and hypercapnia conditions was statistically significant $(p<0.05)$.

Positive dynamics was observed in the cardiovascular system; statistically significant increases were noted in both groups for heart rate at rest, BPd after exercise, BPp after exercise, and recovery time of heart rate and $\mathrm{BP}$.

With regard to the physical load tolerance in the 6MWD test, there were statistically significant increases in MG patients $(p<0.05)$; in CG, the values tended to increase but did not reach a statistically significant difference $(p>0.05)$ (Figure 2$)$. 


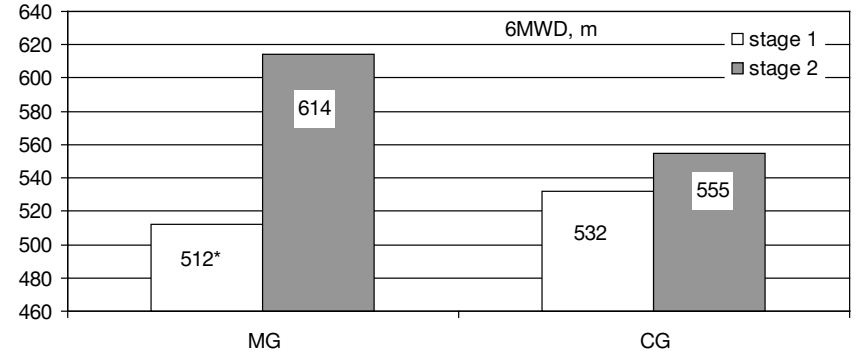

* statistically significant difference in MG $(p<0.05)$ 6MWD - 6-minute walk distance, MG - main group, $\mathrm{CG}$ - control group

Figure 2. Dynamics of daily load tolerance in patients of both groups

Thus, the results of the study showed a statistically significant dynamics of the parameters of the respiratory and cardiovascular systems in MG patients after applying the authors' program of physical rehabilitation. In CG, a positive trend was observed, but statistical significance was not reached in all indicators.

\section{Discussion}

The coordinated statement of the European Respiratory Society and the American Thoracic Society declares that pulmonary rehabilitation is a multidisciplinary, evidence-based, comprehensive system of measures for a patient with COPD who has a clinically significant course of the disease and a violation of the level of daily activity [7]. In the complex treatment of patients with COPD, pulmonary rehabilitation is introduced into the daily therapy; it reduces the manifestations of the disease, optimizes the functional status of the patient, and lowers the cost of treatment by stabilizing or decreasing the systemic manifestations of the disease. Physical rehabilitation in COPD remains an issue. The current literature provides a description of pulmonary rehabilitation, which should be recommended to all patients apart from the pharmacological treatment [14-17]. Unfortunately, among the published research results on the treatment of the disease, there are 9.8$15.95 \%$ of rehabilitation studies and $3.8-11.5 \%$ of those related to physiotherapy $[16,18]$.

Our study was conducted in the context of existing data on the impact of physical rehabilitation on the condition of COPD patients; it turns out to play a significant role in the treatment and prevention of the disease. Many works are devoted to the use of physiotherapy treatment in addition to drug therapy $[9,10,15,19,20]$. In our opinion, to improve the quality of life of COPD patients, it is necessary to employ daily kinesiotherapy, whose main means is breathing exercises, in combination with nebulizer therapy. Many studies have shown the positive effect of respiratory gymnastics $[10,14,17,19]$ and physical training $[1,3,9]$ on the indicators of external respiration function, namely the increase in forced expiratory volume in 1 second. The results of several randomized controlled studies have revealed that the use of manual therapy with physical training helps to improve physical performance $[5,20]$. Some papers provide research results related to the application of Nordic walking [2, 6, 21], which can be a useful practice for normalizing the work of the cardiovascular system. The use of our comprehensive physical rehabilitation program contributed to statistically significant positive changes in all indicators of respiratory function, slowing the recovery of heart rate and blood pressure and increasing exercise tolerance in middle-aged patients with COPD stage IIB, which points at some development in the economization of the respiratory and cardiovascular systems. The obtained data lead to a conclusion that the program of physical rehabilitation for COPD patients should be based not only on changes in the respiratory system, but also on those concerning the cardiovascular system, level of physical activity, and tolerance of daily exercise.

Unfortunately, works devoted to the complex rehabilitation of COPD patients with the use of various means of physical rehabilitation are insufficient in the scientific literature. Therefore, the purpose of our study was to determine the effectiveness of a physical rehabilitation program aimed at improving the functional state of the respiratory and cardiovascular systems, as well as physical performance in middleaged patients with COPD.

\section{Limitations}

In the presented study, there are some limitations. Firstly, the small number of patients who were involved, including only men, limits the possibility to make generalizations for other groups of the population. Secondly, the study was conducted only within the framework of one institution, which may restrain summarizing the results.

\section{Conclusions}

The findings of the study showed that the proposed physical rehabilitation program for middle-aged patients suffering from COPD stage IIB for use in polyclinic conditions contributed to the development of respiratory system economization, slowing down the recovery of cardiovascular parameters, and improving the tolerance of daily exercise, which was confirmed by mathematical processing of the received data.

\section{Disclosure statement}

No author has any financial interest or received any financial benefit from this research.

\section{Conflict of interest}

The authors state no conflict of interest.

\section{References}

1. Bisca GW, Camillo CA, Cavalheri V, Pitta F, Osadnik CR. Peripheral muscle training in patients with chronic obstructive pulmonary disease: novel approaches and recent advances. Expert Rev Respir Med. 2017;11(5): 413-423; doi: 10.1080/17476348.2017.1317598.

2. Breyer MK, Breyer-Kohansal R, Funk GC, Dornhofer N, Spruit MA, Wouters EF, et al. Nordic walking improves daily physical activities in COPD: a randomised controlled trial. Respir Res. 2010;11:112; doi: 10.1186/1465-992111-112.

3. Coultas DB, Jackson BE, Russo R, Peoples J, Sloan J, Singh KP, et al. A lifestyle physical activity intervention for patients with chronic obstructive pulmonary disease. A randomized controlled trial. Ann Am Thorac Soc. 2016; 13(5):617-626; doi: 10.1513/AnnalsATS.201508-508OC.

4. Dodd JW, Hogg L, Nolan J, Jefford H, Grant A, Lord VM, et al. The COPD assessment test (CAT): response to pulmonary rehabilitation. A multicentre, prospective study. Thorax. 2011;66(5):425-429; doi: 10.1136/thx.2010. 156372.

5. Engel RM, Wearing J, Gonski P, Vemulpad S. The effect of combining manual therapy with exercise for mild chronic obstructive pulmonary disease: study protocol 
for a randomised controlled trial. Trials. 2017;18(1):282; doi: 10.1186/s13063-017-2027-z.

6. Fritz T, Caidahl K, Krook A, Lundström P, Mashili F, Osler M, et al. Effects of Nordic walking on cardiovascular risk factors in overweight individuals with type 2 diabetes, impaired or normal glucose tolerance. Diabetes Metab Res Rev. 2013;29(1):25-32; doi: 10.1002/dmrr.2321.

7. GOLD 2017 Global Strategy for the Diagnosis, Management and Prevention of COPD. Available from: https:// goldcopd.org/gold-2017-global-strategy-diagnosismanagement-prevention-copd/.

8. Holland AE, Hill CJ, Rasekaba T, Lee A, Naughton MT, McDonald CF. Updating the minimal important difference for six-minute walk distance in patients with chronic obstructive pulmonary disease. Arch Phys Med Rehabil. 2010;91(2):221-225; doi: 10.1016/j.apmr.2009.10.017.

9. Jackson BE, Coultas D, Russo R, Ashmore J, Sloan J, Uhm $\mathrm{M}$, et al. Benefits of a lifestyle physical activity intervention for COPD are limited to patients with moderate impairment [abstract]. Am J Respir Crit Care Med. 2015;191:A4448.

10. Mohammed J, Da Silva H, Van Oosterwijck J, Calders P. Effect of respiratory rehabilitation techniques on the autonomic function in patients with chronic obstructive pulmonary disease: a systematic review. Chron Respir Dis.2017;14(3):217-230;doi:10.1177/1479972316680844.

11. Ambrosino N, Bertella E. Lifestyle interventions in prevention and comprehensive management of COPD. Breathe. 2018;14(3):186-194; doi: 10.1183/20734735. 018618.

12. Frisk B, Espehaug B, Hardie JA, Strand LI, Moe-Nilssen R, Eagan TM, et al. Physical activity and longitudinal change in 6-min walk distance in COPD patients. Respir Med. 2014;108(1):86-94; doi: 10.1016/j.rmed.2013.09. 004.

13. Marciniuk DD, Brooks D, Butcher S, Debigare R, Dechman G, Ford G, et al. Optimizing pulmonary rehabilitation in chronic obstructive pulmonary disease - practical issues: a Canadian Thoracic Society Clinical Practice Guideline. Can Respir J. 2010;17(4):159-168; doi: 10.1155/ 2010/425975.

14. McCarthy B, Casey D, Devane D, Murphy K, Murphy E, Lacasse Y. Pulmonary rehabilitation for chronic obstructive pulmonary disease. Cochrane Database Syst Rev. 2015;2:CD003793; doi: 10.1002/14651858.CD003793. pub3.

15. Polastri M, Comellini V, Pacilli AMG, Nava S. Magnetic stimulation therapy in patients with COPD: a systematic review. COPD. 2018;15(2):165-170; doi: 10.1080/15412 555.2018.1439910.

16. Tymruk-Skoropad K, Tsizh L, Vynogradskyi B, Pavlova I. Physical therapy in chronic obstructive pulmonary disease (analysis of the evidence-based medicine). Physiother Quart. 2018;26(2):1-8; doi: 10.5114/pq.2018.75993.

17. Wu W, Guan L, Zhang X, Li X, Yang Y, Guo B, et al. Effects of two types of equal-intensity inspiratory muscle training in stable patients with chronic obstructive pulmonary disease: a randomised controlled trial. Respir Med. 2017;132:84-91; doi: 10.1016/j.rmed.2017.10.001.

18. Waschki B, Kirsten AM, Holz O, Mueller KC, Schaper M, Sack AL, et al. Disease progression and changes in physical activity in patients with chronic obstructive pulmonary disease. Am J Respir Crit Care Med. 2015;192(3):295306; doi: 10.1164/rccm.201501-00810C.
19. Langer D, Charususin N, Jácome C, Hoffman M, McConnell $A$, Decramer $M$, et al. Efficacy of a novel method for inspiratory muscle training in people with chronic obstructive pulmonary disease. Phys Ther. 2015;95(9):12641273; doi: 10.2522/ptj.20140245.

20. López-García A, Souto-Camba S, Blanco-Aparicio M, González-Doniz L, Saleta JL, Verea-Hernando H. Effects of a muscular training program on chronic obstructive pulmonary disease patients with moderate or severe exacerbation antecedents. Eur J Phys Rehabil Med. 2016; 52(2):169-175.

21. Stansky NT, Alekseenko AA, Koloshkina VA. Basics of the Nordic walking methodology: methodical recommendations [in Russian]. Vitebsk: Vitebsk State University named after P.M. Masherov; 2015. 